\title{
New $\lambda$ Bootis stars with a shell
}

\author{
B. Hauck ${ }^{1}$, D. Ballereau ${ }^{2}$, and J. Chauville ${ }^{2}$ \\ 1 Institut d'Astronomie de l'Université de Lausanne, CH-1290 Chavannes-des-Bois, Switzerland \\ 2 Observatoire de Paris-Meudon, DASGAL, F-92125 Meudon Principal Cedex, France
}

Received February 26; accepted August 5, 1997

\begin{abstract}
We publish here the second part of our spectroscopic survey at high dispersion of some known and suspected $\lambda$ Bootis stars with a view to detecting circumstellar shell features. Eight stars of our sample exhibit such features. These stars are fast rotators, a result which is in line with Hohlweger and Rentzsch-Holm's study (1995). The analysis of the photometric data has allowed us to confirm the exclusion of a few stars misclassified from the $\lambda$ Bootis group.
\end{abstract}

Key words: stars: chemically peculiar — stars: circumstellar matter — stars: rotation

\section{Introduction}

We have undertaken a spectroscopic survey at high dispersion of some known $\lambda$ Bootis stars with a view to testing the hypothesis of Venn \& Lambert (1990), who suggested a depleted gas accretion model. In a first paper we found (Hauck et al. 1995, hereafter Paper I) circumstellar shell (CS) features in the CaII-K line of two stars (HD 16955 and HD 204965) and a possible such feature for HD 220061. New observations were made at the HauteProvence Observatory in 1994 and 1995 and we present here evidence for the presence of circumstellar components around some other $\lambda$ Bootis stars.

Since publication of Paper I, many papers on the $\lambda$ Bootis stars have been published, among which we may mention those from Holweger \& Rentzsch-Holm (1995) and Andrillat et al. (1995). Holweger and Rentzsch-Holm report on high-resolution observations of the CaII-K line in two samples of A stars, $\lambda$ Bootis and dusty normal A stars, finding CS components for five out of eleven $\lambda$ Bootis stars. Their conclusion is that the presence of detectable amounts of circumstellar gas in A stars is rare among chemically normal A stars. However the $\lambda$ Bootis stars are metal-deficient and nearly half of their sample are surrounded by circumstellar gas. A similar conclusion

Send offprint requests to: B. Hauck is reached by Andrillat et al. (1995), who observed a sample of $20 \lambda$ Bootis stars in three wavelength regions in the near infrared. Seven stars of this sample exhibit evidence of shells. It is also important to pay attention to temporal variations of $V_{\mathrm{r}}$ for both the stellar and circumstellar lines. Many authors have reported lately on the pulsation and variability of some $\lambda$ Bootis stars (Weiss et al. 1994; Kuschnig et al. 1994a,b; Paunzen et al. 1995a,b; Paunzen 1995; Bohlender et al. 1996) and Holweger \& RentzschHolm (1995) have mentioned a $V_{\mathrm{r}}$ variation of the CS component of HD 111786 between 1990 and 1994. At the earlier date the CS component is slightly blue shifted, while at the second date it is red shifted. Faraggiana et al. (1997) consider this star to be a spectroscopic binary whose $\lambda$ Bootis primary has a high rotation speed, while the secondary has an F type and a slow rotation; one of the two components pulsates.

\section{Observations and reduction}

\subsection{Observations}

The first difficulty we encounter in a study on the $\lambda$ Bootis stars is that of establishing a list of candidates. Not all the $\lambda$ Bootis classifications are based on the same criteria, although Gray (1988) has proposed a clear working definition. The stars selected for our survey come from those recognised as being $\lambda$ Bootis stars according to spectroscopic criteria. Our first sources were Gray (1988) and Gray \& Garrison (1987, 1989a,b). We subsequently used three other papers by Abt (1984), Abt \& Morrell (1995) and Andrillat et al. (1995). Such a choice can obviously reveal some disagreement between these authors, our Table 1 showing that many stars considered as $\lambda$ Bootis by Abt or Abt and Morrell are not classified $\lambda$ Bootis by Gray and Garrison.

According to Gray (1988) some stars were classified in the literature as $\lambda$ Bootis on the basis of a weak MgII $\lambda 4481$ line, but some other groups of stars display the same feature, as for example many Ap and helium-weak stars. Thus we can understand why some stars classified $\lambda$ Bootis by Abt are classified Ap by other authors. 
The above discussion shows the difficulty in selecting real $\lambda$ Bootis stars. Some authors (Baschek et al. 1984; Faraggiana et al. 1990) have used UV criteria, but due to the small number of stars in their sample it is not sure if they really are a general property of $\lambda$ Bootis stars. Faraggiana (1987) well resumed the situation before the work of Gray (1988), while Gerbaldi \& Faraggiana (1993) have produced a list of what we could call real $\lambda$ Bootis stars. However, such a list would now need to be revised by adding new stars.

It may be seen from Table 1 of Gerbaldi \& Faraggiana (1993) that among the stars of our Table 1 the following are considered to be real $\lambda$ Bootis stars: HD 31295, HD 38545, HD 110411, HD 111604, HD 125162 and HD 221756. A survey of the literature allows us to propose some more stars: HD 36496, HD 39283 from Andrillat et al. (1995), while HD 217782 was proposed by Parenago (1958) as a $\lambda$ Bootis star and this property was confirmed by Andrillat et al.

It will be seen in Sect. 3 that we propose the exclusion of four stars from the $\lambda$ Bootis group: HD 47152, HD 108283, HD 204965 and HD 225180. HD 159082 and HD 196821 are also classified as HgMn stars and, taking into account the remark of Gray (1988), they may also be excluded. The status of HD 112097 is not very clear. This star is classified as Am by Levato \& Abt (1978) and is classified F0Vp ( $\lambda$ Bootis, met: A7) by Abt \& Morrell (1995). Photometric data (see Sect. 3) agree with $\lambda$ Bootis type, this star being slightly metal-deficient. HD 110377 is found to be a $\delta$ Sct star by Peniche et al. (1981).

The remaining stars have either an alternate classification or are classified only by Abt (1984) or Abt \& Morrell (1995). Gray \& Garrison (1987, 1989a,b) have classified the following stars as normal, while Abt or Abt and Morrell classified them as $\lambda$ Bootis: HD 11503, HD 16811, HD 30739, HD 118623, HD 125489, HD 153808, HD 161868, HD 210418, HD 214454 and HD 220061.

HD 34787 is classified as $\lambda$ Bootis by Abt \& Morrell (1995), while Faraggiana et al. (1990) have excluded it. Finally HD 2904, HD 5789 and HD 109980 are classified as $\lambda$ Bootis only by Abt and Morrell and HD 141851 by Abt. Such an enumeration of disagreement clearly shows the necessity for a critical survey by a spectroscopic specialist of all $\lambda$ Bootis stars and $\lambda$ Bootis candidates. Since the submission of this paper, a consolidated catalogue of $\lambda$ Bootis stars has been lately published (Paunzen et al. 1997) and surely it could play this role.

Some of these stars are already mentioned in the literature as having circumstellar matter. They are HD 38545 (Stürenburg 1993; Bohlender \& Walker 1994, Paper I), HD 39283 (Andrillat et al. 1995), HD 108283 (Jaschek et al. 1991) and HD 217782 (Andrillat et al. 1995). Concerning HD 31295, HD 110411 and HD 221756, Stürenburg (1993) mention the presence of a marginal shell. For some stars of our sample an IR excess is mentioned in the literature. King (1994) has found such an excess for HD 31295, HD 125162 and HD 161868 and a marginal excess for HD 111604. Cheng et al. (1995) find an IR excess for HD 110411.

Our observations were made during four sessions at the Haute-Provence Observatory in November 1994, February 1995, May 1995 and October-November 1995, using the $1.52 \mathrm{~m}$ telescope equipped with the AURELIE spectrograph. The detector is a double-element TH7832 with sets of 2048 photodiodes of $750 \times 13 \mathrm{~mm}$. Two gratings were used ( $\mathrm{N}^{\circ} 7$ with 1800 lines $/ \mathrm{mm}$ and $\mathrm{N}^{\circ} 2$ with 1200 lines $/ \mathrm{mm}$ ). More details on AURELIE can be found in Gillet et al. 1994).

Table 1 lists the stars observed during the four sessions, together with some of their characteristics. The fourth column, $\mathrm{S} 1$, gives the source of $\lambda$ Bootis classification. Spectral types are from Abt and Morrell (Col. 6) and Gray and Garrison (Col. 7). Radial velocities are from the Bright Star Catalogue (Hoffleit \& Jaschek 1982), while $V \sin i$ is taken from Abt \& Morrell (1995) and photometric data, including $m_{v}$, are taken from the Geneva Database. Table 2 gives a record of the new observations, the latter being made in the following spectral regions: $\mathrm{H} \gamma$, CaII-K, NaI-D and for a few stars, at $\mathrm{H} \alpha$ and $\mathrm{H} \beta$.

\subsection{Radial velocities of photospheric lines}

As in Paper I, we have taken advantage of an excellent signal-to-noise ratio to determine the radial velocity of the main photospheric lines ( $\mathrm{H} \alpha, \mathrm{H} \beta, \mathrm{H} \gamma, \mathrm{H}_{8}$ and CaII-K). It is recalled that two procedures were used to derive the radial velocity. Each line was measured twice: by parabolic fit from the bottom of the profile and by the gravity centre of its lower part, limited by a straight horizontal line. Table 3 gives these two measurements for each observed line. In this table, radial velocities are given to one decimal digit, for the following reason. The wavelength of the line's bottom is defined by the minimum of the parabola fitted on $N \pm 7$ point in the line core, and its precision is given approximately by the formula:

$$
\Delta X \simeq \frac{2}{\sqrt{N-2}} \frac{\text { sampling step }}{\mathrm{S} / \mathrm{N}}
$$

obtained by a similar way as the formula given by Gerstenkorn et al. (1977). With a $0.1 \AA$ step for grating $\mathrm{N}^{\circ} 2$ and $S / N \geq 300$, we therefore obtain $\Delta X \simeq 3$ $10^{-4} \AA$, which is lower than the error of the Th-Ar dispersion curve, $0.015 \AA$. For a $\lambda$ of $4000 \AA$ (Ca II), this represents, in RV of the parabolic fit, a precision of $\sim$ $1 \mathrm{~km} \mathrm{~s}^{-1}$, thus justifying the 1-decimal digit of RVs in Table 3.

In general, the radial velocities of the hydrogen lines are in agreement, although they are sometimes different from those of CaII-K. In Paper I, we suggested that these 
differences may be explained either by the different velocities of the hydrogen winds and of ionised calcium (stratification of the elements) or by the presence of a binary system whose spectral components vary in phase opposition, the hottest component showing $\mathrm{H}$-lines and the coolest Ca II-K line. In some cases our mean radial velocities show a marked difference with those given by Hoffleit \& Jaschek (1982). We will revert to these points in Sect. 5 .

\subsection{Radial velocities of shell lines}

All the line profiles were examined carefully to detect a CS component. In addition to HD 16955 and HD 204965, already discussed in Paper I, we found eight more stars with such a component. Figure 1 shows the profile of the CaII-K line for these stars. It may be noted that no clearly pronounced CS component was found for HD 39283, while Andrillat et al. (1995) consider this star as LB shell. The radial velocity and the equivalent width of the CS component were then determined. Table 4 gives for each star these two quantities.

We obtained a spectrum of the CaII-K line of the $\lambda$ Bootis prototype star (HD 125162), which deserves special mention. Barring errors on our part, no CaII-K line profiles of HD 125162 have been published up to the present.

Figure 2 shows this line, between $\mathrm{H}_{\epsilon}$ and $\mathrm{H}_{8}$, for $\lambda$ Boo and HD 31295 ( $\pi^{1}$ Ori), which has the same spectral type $(\mathrm{A} 0 \mathrm{~V})$ and the same projected rotation velocity $\left(V \sin i=105 \mathrm{~km} \mathrm{~s}^{-1}\right)$. The equivalent CaII-K widths are respectively:

$W_{\mathrm{CaII}-\mathrm{K}}=400.8 \mathrm{~m} \AA(\lambda$ Boo $)$

$W_{\text {CaII-K }}=637.6 \mathrm{~m} \AA$ (HD 31295).

Figure 3 shows an enlargement of the $\lambda$ Boo CaII-K line illustrated in Fig. 2.

Contrary to what one would expect, this star has no circumstellar component in fine absorption, but what would appear to be two slight components in emission. It will be seen that at the bottom of the CaII-K line of $\lambda$ Boo two weak emissions that are well separated from each other appear above the photospheric profile. They may be compared with what appears on the $\mathrm{H}_{\gamma}$ line of the Be star HD 47054 in Ballereau et al. 1995 (p. 440). However, this emission was only observed on one profile and has to be confirmed by further observations.

\section{Photometric data}

Data for Table 1 are taken from the Geneva Database maintained by G. Burki. B2-V1 is a temperature parameter, $d$ a luminosity parameter, $\Delta m_{2}$ a blanketing parameter and $\Delta$ a parameter sensitive to luminosity and blanketing. Before using B2-V1 to determine $T_{\text {eff }}$, we have to consider if the stars are reddened. For this we can first of all use the Strömgren photometry and then the code developed by Künzli et al. (1997). Only HD 225180 was detected as being reddened.

As regards a luminosity effect on B2-V1, Hauck (1985) has shown that as far as classes V to III are concerned the $T_{\text {eff }}$ vs. B2-V1 relation is not influenced by luminosity effect. $T_{\text {eff }}$ was derived from a relation between $T_{\text {eff }}$ and B2-V1 (Hauck 1994). The precision of such a $T_{\text {eff }}$ is of order of $\pm 200 \mathrm{~K}$. Hauck \& Künzli (1996) have shown that the $T_{\text {eff }}$ determined by the relation between $T_{\text {eff }}$ and B2-V1 have an accurency of the same order as those determined by the code of Künzli et al. (1997), i.e. \pm 200 K. Since many $\lambda$ Bootis stars have a high $V$ sin $i$ value it should be recalled that B2-V1 is affected by such high values (Hauck $\&$ Slettebak 1989). Stars with high $V \sin i$ values have too red a B2-V1 value. For A1 stars for instance, the mean $\mathrm{B} 2-\mathrm{V} 1$ value for $V \sin i$ values between 0 and 100 is 0.134 while it is -0.100 for $V \sin i$ values between 200 and 400 . Thus for the latter stars the $T_{\text {eff }}$ value given in Table 1 is only an estimate of the real $T_{\text {eff }}$.

Since the $\lambda$ Bootis stars are metal-deficient, it is interesting to use the properties of the Geneva system to consider whether the stars of our sample meet this assumption. $\Delta m_{2}$, the difference between $m_{2}$ (stars) $m_{2}$ (Hyades), is a blanketing parameter, well-correlated to $[\mathrm{Fe} / \mathrm{H}]$ (Hauck 1978). Stars with a negative $\Delta m_{2}$ value have a lower $[\mathrm{Fe} / \mathrm{H}]$ than the Hyades stars. According to $\Delta m_{2}$, the following stars are metal-deficient: HD 36496, HD 110377, HD 111604, HD 141851, a few others being only slightly metal-deficient $\left(-0.028 \leq \Delta m_{2} \leq-0.018\right)$.

Some other stars possess interesting photometric properties:

HD 108283 has a positive value of $\Delta m_{2}$ (of the same order as an Am or Ap star) and we may assume that this star does not belong to the $\lambda$ Bootis group. It is mentioned as Sr? by Renson (1992), classified as A9 IVnp Sr II by Gray \& Garrison (1989) but A9 Vp ( $\lambda$ Boo) by Abt \& Morrell (1995). Jaschek et al. (1991) found a moderate shell for this star. We have also found shell components for the same star.

HD 47152 is a well-known $\mathrm{Hg}$ star (Osawa 1965) and this is confirmed by its positive value of the $\Delta(\mathrm{V} 1-\mathrm{G})$ parameter.

HD 204965 has high $d$ and $\Delta$ values and thus in a $d$ vs. $\Delta$ diagram it is located in the region of luminosity class III stars. Iliev \& Barzova (1995) have recently confirmed the assumption that $\lambda$ Bootis stars are main-sequence stars. So we may wonder if HD 204965 belongs to the $\lambda$ Bootis group. We can also reach the same conclusion for HD 225180, which is classified by Gray \& Garrison (1987) as A1 II-III and this is confirmed by our $\mathrm{d}$ and $\Delta$ parameters. This star is reddened, therefore we cannot derive a $\Delta m_{2}$ value.

Thus, on the basis of the photometric data, we can exclude these four stars from the $\lambda$ Bootis group. 
Table 1. General data for the observed stars

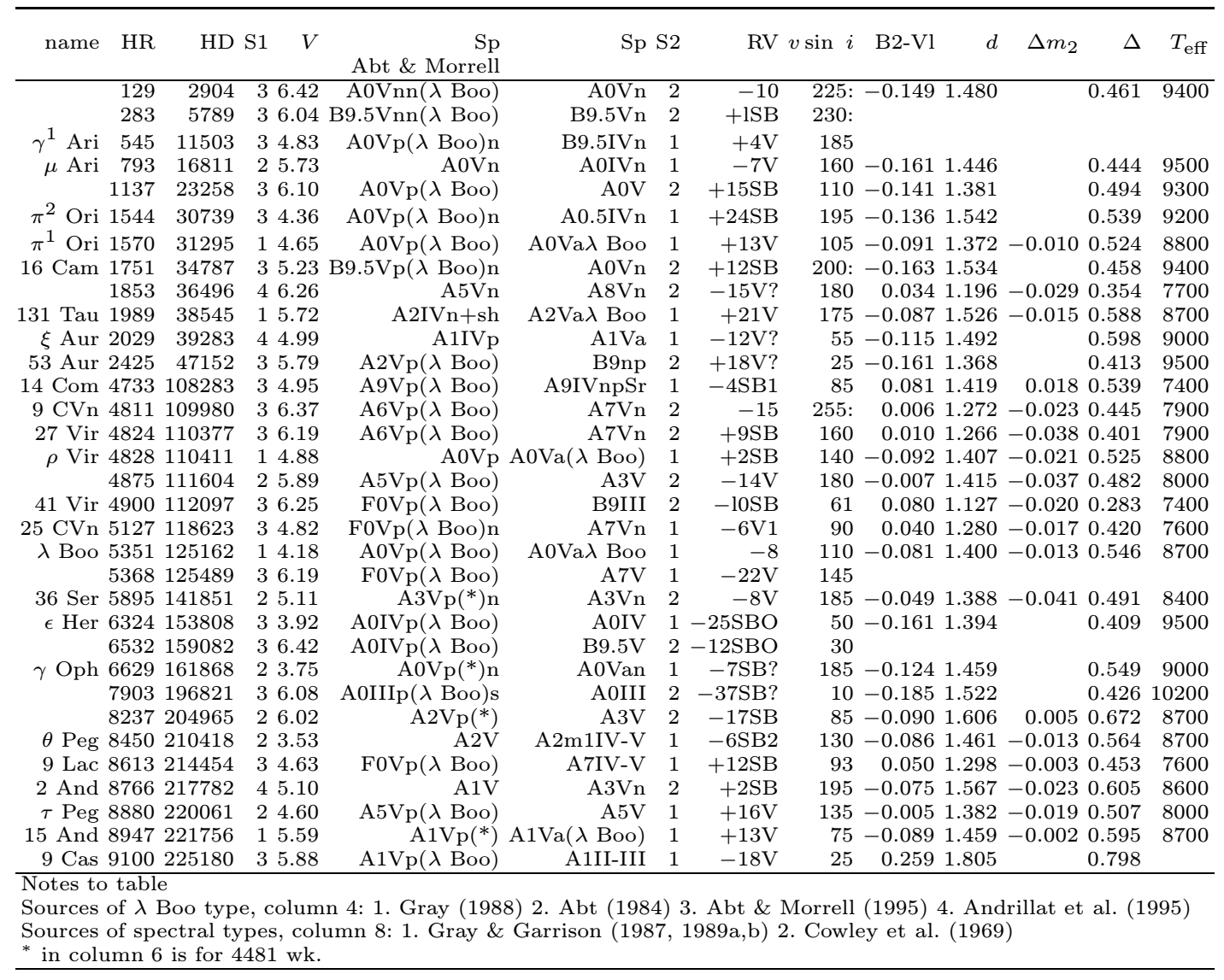

\section{Stars with circumstellar component}

As mentioned in Sect. 2.3, we have found eight stars, in addition to HD 16955 and HD 204965 already discussed in Paper I, presenting a CS component and we have used a relation proposed by Higurashi \& Hirata (1978) to derive the extension of the shell. This relation is:

$r / r^{*}=\left(\Delta V_{1 / 2} / V \sin i\right)^{1 /(1+j)}$

where $r / r *$ is the extension of the shell in stellar radius, $\Delta V_{1 / 2}$ the half-width at half-depth and $j$ a parameter depending from the rotation law of the shell gas. In our case, keplerian rotation, $j=1 / 2$.

We have applied it to the stars of our sample with a CS component, Table 4 showing the results of our calculations.

In addition to the stars of Table 4, we have found two stars for which the presence of a CS component is less marked: HD 39283 and HD 108283. On the basis of its photometric data HD 108283 is not a $\lambda$ Bootis star but an Am or Ap star. No evidence has been found for a CS component in the case of HD 31295 and HD 110411, whereas Sturenburg (1993) finds a marginal CS compo- nent for both of these stars. For HD 110411, Holweger \& Rentzsch-Holm (1995) find a very weak CS component.

The presence of a CS component in the stars of Table 4 is confirmed by some other studies. Andrillat et al. (1995) also find a CS component for HD 38545 and HD 217782. Furthermore, Sturenburg (1993) and Bohlender \& Walker (1994) indicate the presence of a CS component for HD 38545. The fact that some authors find a CS component and others do not is perhaps due to a temporal variation of this CS component. In September 1993, we noticed such a CS component in the spectrum of HD 204965 and in October 1995 this component had disappeared.

Several of our programme stars display the sodium doublet. These stars are listed in Table 5, which includes some of their spectroscopic characteristics and the extension of the circumstellar shell, for which the relation of Higurashi \& Hirata (1978) was used, as in the case of CaII$\mathrm{K}$. Five of the stars in Table 5 possess a CS component on the CaII-K line.

The 14 other stars observed in the NaID region do not display the NaID doublet, either because it is too weak or because it is completely masked by the telluric lines which overcrowd this spectral domain. 
Table 2. Log of observations (1994-1995)

\begin{tabular}{|c|c|c|c|c|c|c|}
\hline $\begin{array}{l}\text { star } \\
\text { HD }\end{array}$ & line $(s)$ & $\begin{array}{r}\text { date } \\
\mathrm{d} / \mathrm{m} / \mathrm{y}\end{array}$ & HJD-2400000 & $\begin{array}{r}\text { exposure } \\
\text { time (min.) }\end{array}$ & resolution & $\mathrm{S} / \mathrm{N}$ \\
\hline 2904 & $\mathrm{H}_{8}-\mathrm{CaIIK}$ & $01 / 11 / 95$ & 50023.480 & 158 & 14000 & 180 \\
\hline \multirow[t]{2}{*}{5789} & $\mathrm{H}_{8}$-CaIIK & $31 / 10 / 95$ & 50022.417 & 105 & 14000 & 450 \\
\hline & NaID & $02 / 11 / 95$ & 50024.469 & 120 & 21000 & 320 \\
\hline \multirow[t]{2}{*}{11503} & $\mathrm{H}_{8}-\mathrm{CaIIK}$ & $10 / 11 / 94$ & 49667.420 & 43 & 23000 & 380 \\
\hline & $\mathrm{H}_{\gamma}$ & $11 / 11 / 94$ & 49668.389 & 47 & 25000 & 470 \\
\hline \multirow[t]{2}{*}{16811} & $\mathrm{H}_{8}-\mathrm{CaIIK}$ & $30 / 10 / 95$ & 50021.474 & 76 & 14000 & 390 \\
\hline & NaID & $03 / 11 / 95$ & 50025.419 & 132 & 21000 & 420 \\
\hline \multirow[t]{2}{*}{23258} & $\mathrm{H}_{8}-\mathrm{CaIIK}$ & $01 / 11 / 95$ & 50022.585 & 98 & 14000 & 300 \\
\hline & NaID & 03/11/95 & 50025.551 & 164 & 21000 & 300 \\
\hline 30739 & $\mathrm{H}_{\gamma}$ & $11 / 11 / 94$ & 49668.436 & 66 & 25000 & 430 \\
\hline 31295 & $\mathrm{H}_{\gamma}$ & $11 / 11 / 94$ & 49668.510 & 66 & 25000 & 450 \\
\hline \multirow[t]{4}{*}{34787} & $\mathrm{H}_{8}-\mathrm{CaIIK}$ & $01 / 11 / 94$ & 49667.563 & 62 & 23000 & 350 \\
\hline & $\mathrm{H}_{8}$-CaIIK & $02 / 11 / 95$ & 50023.675 & 108 & 14000 & 280 \\
\hline & $\mathrm{H}_{\gamma}$ & $12 / 11 / 94$ & 49668.556 & 62 & 25000 & 420 \\
\hline & NaID & $03 / 11 / 95$ & 50024.559 & 65 & 21000 & 460 \\
\hline 36496 & $\mathrm{H}_{8}$-CaIIK & $31 / 10 / 95$ & 50021.632 & 120 & 14000 & 270 \\
\hline \multirow[t]{3}{*}{38545} & $\mathrm{H}_{8}-\mathrm{CaIIK}$ & $11 / 11 / 94$ & 49667.630 & 113 & 23000 & 350 \\
\hline & $\mathrm{H}_{\gamma}$ & $12 / 11 / 94$ & 49668.653 & 102 & 25000 & 430 \\
\hline & NaID & $04 / 11 / 95$ & 50025.662 & 150 & 21000 & 300 \\
\hline \multirow[t]{3}{*}{39283} & $\mathrm{H}_{8}-\mathrm{CaIIK}$ & $31 / 10 / 95$ & 50021.694 & 43 & 14000 & 210 \\
\hline & $\mathrm{H}_{\beta}$ * & $05 / 11 / 95$ & 50026.677 & 101 & 17000 & 600 \\
\hline & NaID & $03 / 11 / 95$ & 50024.606 & 64 & 21000 & 480 \\
\hline \multirow[t]{2}{*}{47152} & $\mathrm{H}_{8}-\mathrm{CaIIK}$ & $01 / 11 / 95$ & 50022.656 & 89 & 14000 & 160 \\
\hline & NaID & $03 / 11 / 95$ & 50024.673 & 120 & 21000 & 150 \\
\hline \multirow[t]{2}{*}{108283} & $\mathrm{H}_{8}-\mathrm{CaIIK}$ & $05 / 05 / 95$ & 49843.363 & 96 & 23000 & 350 \\
\hline & NaID* & $08 / 05 / 95$ & 49846.356 & 60 & 34000 & 400 \\
\hline 109980 & $\mathrm{NaID}$ & $10 / 05 / 95$ & 49848.389 & 167 & 34000 & 420 \\
\hline 110377 & NaID & $09 / 05 / 95$ & 49847.383 & 167 & 34000 & 470 \\
\hline \multirow[t]{5}{*}{110411} & $\mathrm{H}_{8}$-CaIIK & $06 / 05 / 95$ & 49844.382 & 111 & 23000 & 450 \\
\hline & $\mathrm{H}_{\gamma}$ & $07 / 02 / 95$ & 49755.582 & 19 & 15000 & 430 \\
\hline & $\mathrm{H}_{\beta}$ & $10 / 02 / 95$ & 49758.608 & 38 & 17000 & 500 \\
\hline & NaID & $08 / 05 / 95$ & 49846.404 & 59 & 34000 & 520 \\
\hline & $\mathrm{H}_{\alpha}$ & $10 / 02 / 95$ & 49758.671 & 23 & 23000 & 380 \\
\hline \multirow[t]{2}{*}{111604} & $\mathrm{H}_{8}$-CaIIK & $07 / 05 / 95$ & 49845.395 & 161 & 23000 & 380 \\
\hline & $\mathrm{H}_{\gamma}$ & $07 / 02 / 95$ & 49755.614 & 46 & 15000 & 420 \\
\hline 112097 & $\mathrm{H}_{\gamma}$ & $07 / 02 / 95$ & 49755.658 & 66 & 15000 & 260 \\
\hline \multirow[t]{4}{*}{118623} & $\mathrm{H}_{8}-\mathrm{CaIIK}$ & $05 / 05 / 95$ & 49843.438 & 77 & 23000 & 400 \\
\hline & $\mathrm{H}_{\gamma}$ & $07 / 02 / 95$ & 49755.688 & 16 & 15000 & 470 \\
\hline & NaID & $08 / 05 / 95$ & 49846.448 & 58 & 34000 & 450 \\
\hline & $\mathrm{H}_{\alpha}$ & $10 / 02 / 95$ & 49758.690 & 27 & 23000 & 420 \\
\hline \multirow[t]{2}{*}{125162} & $\mathrm{H}_{8}-\mathrm{CaIIK}$ & $05 / 05 / 95$ & 49843.490 & 40 & 23000 & 450 \\
\hline & $\mathrm{NaID}$ & $08 / 05 / 95$ & 49846.484 & 23 & 34000 & 480 \\
\hline \multirow[t]{2}{*}{125489} & $\mathrm{H}_{8}$-CaIIK* & $06 / 05 / 95$ & 49844.995 & 300 & 23000 & 370 \\
\hline & $\mathrm{NaID}^{*}$ & $09 / 05 / 95$ & 49847.983 & 187 & 34000 & 360 \\
\hline \multirow[t]{2}{*}{141851} & $\mathrm{H}_{8}$-CaIIK & $06 / 05 / 95$ & 49843.547 & 77 & 23000 & 260 \\
\hline & NaID & $08 / 05 / 95$ & 49846.530 & 91 & 34000 & 480 \\
\hline \multirow[t]{2}{*}{153808} & $\mathrm{H}_{8}$-CaIIK & $06 / 05 / 95$ & 49843.584 & 22 & 23000 & 380 \\
\hline & $\mathrm{NaID}$ & $09 / 05 / 95$ & 49846.568 & 15 & 34000 & 450 \\
\hline 159082 & $\mathrm{H}_{8}$-CaIIK* & $07 / 05 / 95$ & 49845.089 & 225 & 23000 & 300 \\
\hline & NaID & $11 / 05 / 95$ & 49848.564 & 103 & 34000 & 901 \\
\hline 161868 & $\mathrm{H}_{8}-\mathrm{CaIIK}$ & $06 / 05 / 95$ & 49843.607 & 24 & 23000 & 310 \\
\hline & NaID & $09 / 05 / 95$ & 49846.587 & 22 & 34000 & 530 \\
\hline 196821 & $\mathrm{H}_{8}-\mathrm{CaIIK}$ & $31 / 10 / 95$ & 50022.297 & 111 & 14000 & 200 \\
\hline & NaID & $02 / 11 / 95$ & 50024.305 & 120 & 21000 & 220 \\
\hline 204965 & $\mathrm{H}_{8}$-CaIIK & $30 / 10 / 95$ & 50021.328 & 114 & 14000 & 300 \\
\hline 210418 & $\mathrm{H}_{8}$-CaIIK & $10 / 11 / 94$ & 49667.253 & 24 & 23000 & 300 \\
\hline & $\mathrm{H}_{8}-\mathrm{CaIIK}$ & $01 / 11 / 95$ & 50023.277 & 26 & 14000 & 400 \\
\hline & $\mathrm{H}_{\gamma}$ & $11 / 11 / 94$ & 49668.266 & 51 & 25000 & 360 \\
\hline
\end{tabular}


Table 2. continued

\begin{tabular}{rrrrrrr}
\hline $\begin{array}{r}\text { star } \\
\mathrm{HD}\end{array}$ & line $(\mathrm{s})$ & $\begin{array}{r}\text { date } \\
\mathrm{d} / \mathrm{m} / \mathrm{y}\end{array}$ & HJD-2400000 & $\begin{array}{r}\text { exposure } \\
\text { time (min.) }\end{array}$ & resolution & $\mathrm{S} / \mathrm{N}$ \\
\hline 214454 & $\mathrm{H}_{8}$-CaIIK & $10 / 11 / 94$ & 49667.290 & 64 & 23000 & 300 \\
& $\mathrm{H}_{\gamma}$ & $11 / 11 / 94$ & 49668.307 & 54 & 25000 & 300 \\
217782 & $\mathrm{H}_{8}$-CaIIK & $31 / 10 / 95$ & 50022.359 & 48 & 14000 & 380 \\
& $\mathrm{NaID}$ & $03 / 11 / 95$ & 50025.341 & 82 & 21000 & 450 \\
220061 & $\mathrm{H}_{8}$-CaIIK & $10 / 11 / 94$ & 49667.357 & 102 & 23000 & 250 \\
& $\mathrm{H}_{8}$-CaIIK & $01 / 11 / 95$ & 50023.310 & 55 & 14000 & 380 \\
& $\mathrm{H}_{\gamma}$ & $11 / 11 / 94$ & 49668.349 & 51 & 25000 & 300 \\
221756 & $\mathrm{H}_{8}$-CaIIK & $01 / 11 / 95$ & 50023.373 & 120 & 14000 & 400 \\
225180 & $\mathrm{NaID}$ & $02 / 11 / 95$ & 50024.388 & 100 & 21000 & 520 \\
& $\mathrm{H}_{8}$-CaIIK & $30 / 10 / 95$ & 50021.407 & 98 & 14000 & 300 \\
& $\mathrm{NaID}$ & $04 / 11 / 95$ & 50026.354 & 300 & 21000 & 400 \\
\hline \multicolumn{7}{c}{$* .$. mean of two spectra (HJDs are averaged, and exposure times added). } \\
\hline \multicolumn{7}{c}{}
\end{tabular}

A comparison of the RVs of the CaII-K CS component (Table 4) with the NaI doublet (Table 5) reveals that they are always similar to each other. It may be assumed that these ions are associated in the expansion/contraction movement of the circumstellar shell. It is also noted that the absorption shell of NaI is inside that of CaII. Special mention is made of the sodium doublet of HD 225180, shown in Fig. 4. These lines are strong and all the lines of the optical spectrum possess a shell component. The NaI doublet presents a double structure in which a photospheric and a red-shift shell component may be identified. The shell component has an asymmetric background.

The RVs of the points of inflexion on each blue edge are $-39.5 \mathrm{~km} \mathrm{~s}^{-1} 1(5890 \AA)$ and $-36.0 \mathrm{~km} \mathrm{~s}^{-1}(5896 \AA)$ respectively. Close to the stellar continuum, at $I=0.96$, the total width of each line is $83.2 \mathrm{~km} \mathrm{~s}^{-1}$ and $77.7 \mathrm{~km} \mathrm{~s}^{-1}$ and the RV line at this level is -21.0 and $-20.7 \mathrm{~km} \mathrm{~s}^{-1}$.

Finally, the velocity gradient along each line is $+12.6 \mathrm{~km} \mathrm{~s}^{-1}$ and $+12.1 \mathrm{~km} \mathrm{~s}^{-1}$ respectively, indicating a circumstellar movement towards the star (onfall).

\section{Discussion}

\subsection{Difference of $V_{\mathrm{r}}$ between $H_{8}$ and CaII-K lines}

As noticed in Paper I, we may remark in some cases a difference between the radial velocity deduced from $\mathrm{H}_{8}$ and that from CaII-K. Our sample is now sufficiently large to see clearly from the data of Table 3 that this is not a general property since nearly half of the sample does not exhibit such a gradient, including $\lambda$ Boo itself. Moreover, no correlation has been found between the presence or absence of this gradient and one of the criteria, or suspected criteria, such as the IR excess or UV depression. However as can be seen from Table 1, nearly all the stars studied are spectroscopic binaries or have a variable radial velocity. Thus we can assume that this difference is due to the binarity of these stars.

\subsection{Variation of $V_{\mathrm{r}}$ between two periods of observations}

A variation of $V_{\mathrm{r}}$ between two or more periods of observations could be an indication of binarity or of pulsation. There are too few measurements for a clear conclusion to be reached from our observations. We can only remark that of the six stars for which we have data in the same spectral regions at two or three epochs only one (HD 204965) exhibits a severe change of radial velocity in one year and one (HD 38545) a moderate change, while the other four (HD 31295, HD 34787, HD 210418 and HD 220061) are seen to undergo no, or only a small, change.

\subsection{Difference of $V_{\mathrm{r}}$ between the photospheric lines and the circumstellar lines}

We can consider the difference of $V_{\mathrm{r}}$ between the photospheric line and the circumstellar line for stars for which a circumstellar component has been detected on the CaII-K line. This difference may be interpreted as being the radial component of the circumstellar gas movement. This gradient is positive for the following stars, indicating a fall of the gas on the star: HD 2904, HD 16811. For the following stars the gradient is negative, indicating an expansion of the gas: HD 5789, HD 34787 (in 1994 and 1995), HD 141851, HD 161868, HD 217782. The gradient is slightly negative for HD 38545. Two stars of the 1993 mission that also show an onfall, HD 16955 and HD 204965, can be added to the first of the above-mentioned group.

\subsection{Rotation velocities of $\lambda$ Bootis stars with a CS component}

Holweger \& Rentzsch-Holm (1995) have shown that the $\lambda$ Bootis stars with detectable CS gas are to be found preferably among rapid rotators. This is still true if we consider our results. With the exception of HD 204965 and HD 221756, all the stars of Table 4 possess $V \sin i$ 
Table 3. Radial velocities $\left(\mathrm{km} \mathrm{s}^{-1}\right)$ of photospheric lines (The $\mathrm{RV}$ value is taken from the BS Catalogue)
Table 3. continued

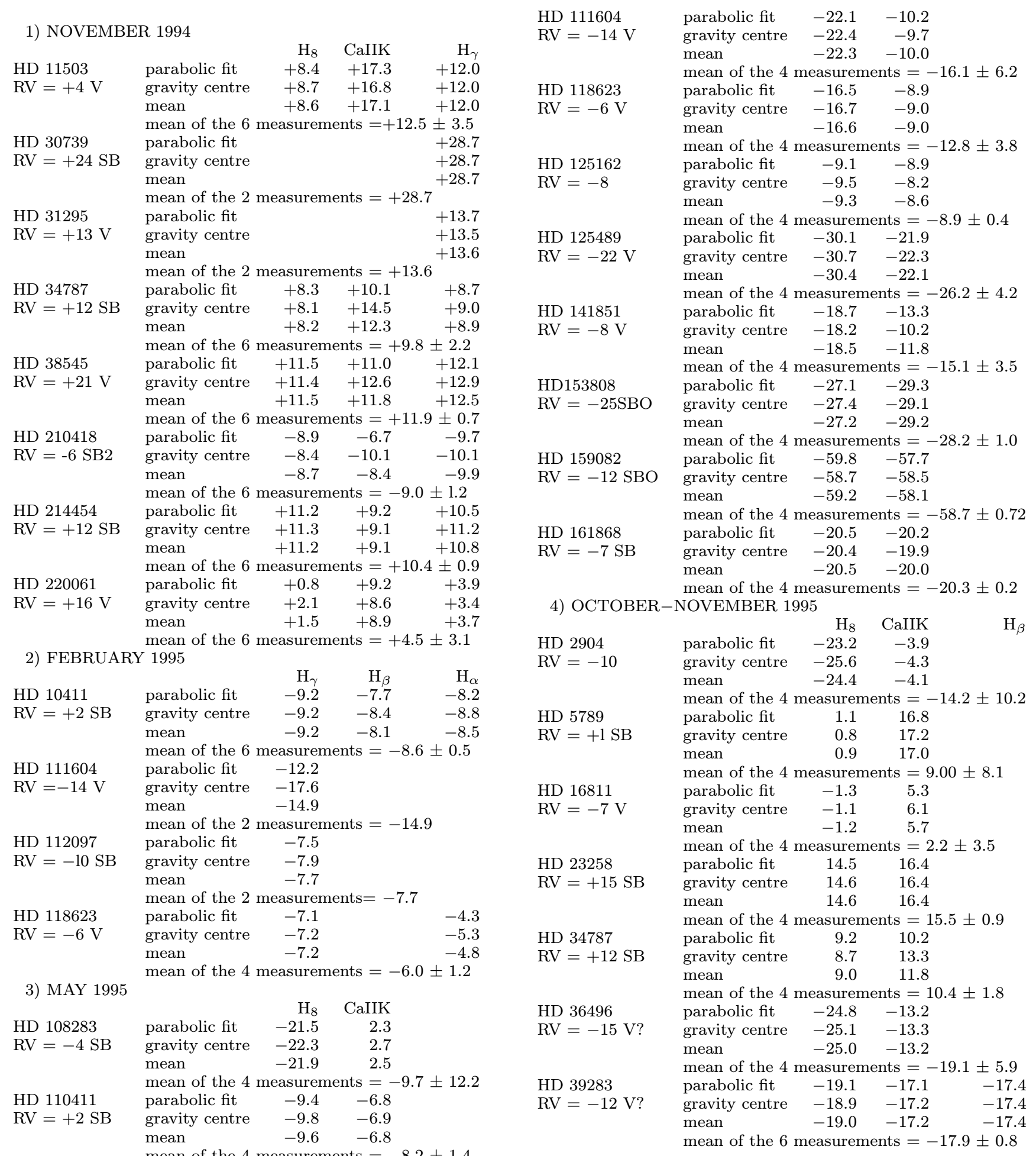


Table 3. continued

\begin{tabular}{|c|c|c|c|}
\hline HD 47152 & parabolic fit & 14.5 & 11.7 \\
\hline \multirow[t]{3}{*}{$\mathrm{RV}=+18 \mathrm{~V} ?$} & gravity centre & 14.6 & 11.8 \\
\hline & mean & 14.5 & 11.8 \\
\hline & \multicolumn{3}{|c|}{ mean of the 4 measurements $=13.1 \pm 1.4$} \\
\hline HD 196821 & parabolic fit & -31.8 & -24.5 \\
\hline \multirow[t]{3}{*}{$\mathrm{RV}=-37 \mathrm{SB} ?$} & gravity centre & -31.8 & -24.2 \\
\hline & mean & -31.8 & -24.4 \\
\hline & \multicolumn{3}{|c|}{ mean of the 4 measurements $=-28.1 \pm 3.73$} \\
\hline HD 204965 & parabolic fit & -16.1 & -14.4 \\
\hline \multirow[t]{3}{*}{$\mathrm{RV}=-17 \mathrm{SB}$} & gravity centre & -16.7 & -14.8 \\
\hline & mean & -16.4 & -14.6 \\
\hline & \multicolumn{3}{|c|}{ mean of the 4 measurements $=-15.5 \pm 0.9$} \\
\hline HD 210418 & parabolic fit & -9.1 & -5.4 \\
\hline \multirow[t]{3}{*}{$\mathrm{RV}=-6 \mathrm{SB} 2$} & gravity centre & -8.9 & -4.8 \\
\hline & mean & -9.0 & -5.1 \\
\hline & \multicolumn{3}{|c|}{ mean of the 4 measurements $=-7.1 \pm 1.9$} \\
\hline HD 217782 & parabolic fit & -3.4 & 3.2 \\
\hline \multirow[t]{3}{*}{$\mathrm{RV}=+2 \mathrm{SB}$} & gravity centre & -4.2 & 2.6 \\
\hline & mean & -3.8 & 2.9 \\
\hline & \multicolumn{3}{|c|}{ mean of the 4 measurements $=-0.5 \pm 3.36$} \\
\hline HD 220061 & parabolic fit & 2.7 & 9.5 \\
\hline \multirow[t]{3}{*}{$\mathrm{RV}=+16 \mathrm{~V}$} & gravity centre & 3.4 & 9.3 \\
\hline & mean & 3.1 & 9.4 \\
\hline & \multicolumn{3}{|c|}{ mean of the 4 measurements $=6.2 \pm 3.2$} \\
\hline HD 221756 & parabolic fit & 12.9 & 14.2 \\
\hline \multirow[t]{3}{*}{$\mathrm{RV}=+13 \mathrm{~V}$} & gravity centre & 13.2 & 14.5 \\
\hline & mean & 13.0 & 14.3 \\
\hline & \multicolumn{3}{|c|}{ mean of the 4 measurements $=13.7 \pm 0.7$} \\
\hline HD 225180 & parabolic fit & -21.9 & -14.0 \\
\hline \multirow[t]{2}{*}{$\mathrm{RV}=-18 \mathrm{~V}$} & gravity centre & -22.2 & -14.7 \\
\hline & mean & -22.0 & -14.4 \\
\hline
\end{tabular}

Table 4. Some data from the circumstellar component of CaII K

\begin{tabular}{|c|c|c|c|c|c|}
\hline star HD & date & $V_{\mathrm{r}}\left(\mathrm{km} \mathrm{s}^{-1}\right)$ & $W_{\lambda}(\mathrm{m} \AA)$ & $\Delta V_{1 / 2}$ & $r / r *$ \\
\hline 2904 & $01 / 11 / 95$ & -2.7 & 13.2 & 13.35 & 6.6 \\
\hline 5789 & $31 / 10 / 95$ & -4.1 & 14.1 & 16.3 & 5.8 \\
\hline 16811 & $30 / 10 / 95$ & 8.1 & 19.7 & 15.0 & 4.8 \\
\hline $16955^{(1)}$ & $20 / 09 / 93$ & 2.9 & 9.6 & 7.1 & 8.0 \\
\hline 34787 & $11 / 11 / 94$ & 5.2 & 18.1 & 8.0 & 8.5 \\
\hline 34787 & $02 / 11 / 95$ & 5.4 & 16.0 & 12.15 & 6.5 \\
\hline 38545 & $11 / 11 / 94$ & 11.1 & 72.2 & 9.9 & 6.8 \\
\hline 141851 & $06 / 05 / 95$ & -27.5 & 2.3 & 6.9 & 9.0 \\
\hline 161868 & $06 / 05 / 95$ & -30.3 & 6.0 & 8.95 & 7.5 \\
\hline $204965^{(2)}$ & $20 / 09 / 93$ & -18.2 & 8.1 & 6.9 & 5.3 \\
\hline 217782 & $31 / 10 / 95$ & -9.8 & 20.0 & 13.75 & 5.9 \\
\hline \multicolumn{6}{|c|}{$\begin{array}{l}{ }^{(1)} \text { Star from Paper I }\left(V \sin i=160 \mathrm{~km} \mathrm{~s}^{-1}\right) \\
{ }^{(2)} \text { Star from Paper I }\left(V \sin i=85 \mathrm{~km} \mathrm{~s}^{-1}\right) \text {. The circumstellar } \\
\text { component has vanished on a spectrum dated } 30 / 10 / 95 . \\
V_{\mathrm{r}}=\mathrm{RV} \text { of the component } \\
\Delta V_{1 / 2}=\text { width of the CaII K line at half-depth; } \\
W_{\lambda}(\mathrm{m} \AA)=\text { Equivalent width, reduced to the stellar } \\
\text { continuum } \\
r / r *=\text { Extension of the circumstellar disk, in stellar radii } \\
\text { (see text). }\end{array}$} \\
\hline
\end{tabular}

values that are greater than $100 \mathrm{~km} \mathrm{~s}^{-1}$. However, on the basis of their photometric data, these two stars cannot be considered to be $\lambda$ Bootis stars. It may also be remarked that not all $\lambda$ Bootis stars with a $V \sin i$ value in excess of $100 \mathrm{~km} \mathrm{~s}^{-1}$ exhibit a CS component.

Holweger and Rentzsch-Holm thus conclude that $V \sin i$ is the prime factor responsible for the presence or absence of CS absorption in CaII-K. They also make the assumption that $\lambda$ Bootis stars are pre- main-sequence objects that are still accreting material left over from the protostellar phase. Our discussion in 5.3 shows that it is difficult to confirm or rule out this assumption on the basis of the difference of $V_{\mathrm{r}}$ between the photospheric lines and the circumstellar lines.

\section{Conclusions}

Our observations confirm the presence of CS gas around eight $\lambda$ Bootis stars, more particularly among the most rapid rotators. This is in line with the recent study of Holweger \& Rentzsch-Holm (1995), who conclude that these stars are in the pre-main-sequence phase of evolution. Furthermore, our data show that the intensity of the CS component is variable on a timescale of months or years. As the actual time resolution of the observations does not cover a timescale of days we cannot arrive at a conclusion for this timescale. The analysis of the photometric data confirms that four $\lambda$ Bootis candidates can be excluded from the $\lambda$ Bootis group.

Acknowledgements. This research has made use of the SIMBAD database, operated at the CDS, Strasbourg, France. One of us $(\mathrm{BH})$ is grateful to the Fonds National suisse de la Recherche Scientifique, which gave its support to part of the research.

\section{References}

Abt H.A., 1984, in The MK Process and MK Classification, Garrison R.F. (ed.). David Dunlap Observatory, p. 340

Abt H.A., Morrell N.I., 1995, ApJS 99, 135

Andrillat Y., Jaschek C., Jaschek M., 1995, A\&A 299, 493

Ballereau D., Chauville J., Zorec J., 1995, A\&AS 111, 423

Baschek B., Heck A., Jaschek C., et al., 1984, A\&A 131, 378

Bohlender D.A., Walker G.A.H., 1994, MNRAS 266, 891

Bohlender D.A., Gonzalez J.-F., Kennelly E.J., 1996, A\&A 307, L9

Cheng K.P., Bruhweiler F.C., Kondo Y., Grady C.A., 1992, ApJ 396, L83

Cowley A., Cowley C., Jaschek M., Jaschek C., 1969, AJ 74, 375

Faraggiana R., 1987, Astrophys. Space Sci. 134, 381

Faraggiana R., Gerbaldi M., Böhm C., 1990, A\&A 235, 311

Faraggiana R., Gerbaldi M., Burnage R., 1997, A\&A 318, 21

Gerbaldi M., Faraggiana R., 1993, in ASP Conf. Ser. 44, 368

Gerstenkorn S., Luc P., Perrin A., Chauville J., 1977, A\&A 58, 255

Gillet D., Burnage R., Kohler D., et al., 1994, A\&AS 108, 1

Gray R.O., Garrison R.F., 1987, ApJS 65, 581 
Table 5. Some spectroscopic data from 8 programme stars which display the Na I D lines $\left(\mathrm{km} \mathrm{s}^{-1}\right)$

\begin{tabular}{|c|c|c|c|c|c|c|c|c|c|c|c|}
\hline \multicolumn{5}{|c|}{ Na I 5890} & \multicolumn{7}{|c|}{ Na I 5896} \\
\hline & $R_{v}$ & $d$ & $\Delta V 1_{1 / 2}$ & $R V_{1 / 2}$ & $W$ & $R_{V}$ & $d$ & $\Delta V_{1 / 2}$ & $R V_{1 / 2}$ & $W$ & $r / r *$ \\
\hline HD 5789 & -3.9 & 0.821 & 24.0 & -4.5 & 95.5 & -3.7 & 0.898 & 21.0 & -4.6 & 45.6 & 4.7 \\
\hline HD 16811 & 9.8 & 0.764 & 19.2 & 10.6 & 108.3 & 11.0 & 0.837 & 21.3 & 11.8 & 73.7 & 4.0 \\
\hline HD 34787 & 6.5 & 0.741 & 20.3 & 7.4 & 131.4 & 6.5 & 0.821 & 20.3 & 6.8 & 75.7 & 4.6 \\
\hline HD 38545 & 13.7 & 0.836 & 42.7 & 17.5 & 126.8 & 17.1 & 0.904 & - & - & 74.8 & 2.6 \\
\hline HD 108283 & 0.3 & 0.548 & 43.8 & -0.8 & 355.2 & 2.8 & 0.612 & 46.5 & 6.9 & 398.9 & 2.6 \\
\hline HD 159082 & -20.3 & 0.628 & 12.0 & -20.3 & 92.4 & -20.4 & 0.700 & 12.0 & -20.4 & 81.5 & 1.8 \\
\hline HD 217782 & -7.4 & 0.798 & 24.1 & -8.4 & 110.2 & -7.8 & 0.883 & 24.6 & -7.0 & 59.2 & 4.0 \\
\hline HD 225180 & -8.4 & 0.359 & 26.3 & -8.9 & 433.9 & -8.6 & 0.406 & 25.2 & -9.2 & 378.4 & 1.0 \\
\hline \multicolumn{12}{|c|}{$\begin{array}{l}R_{V}=\text { radial velocity of the deepest point of the line } \\
d=\text { depth of the line, in unit of the continuum intensity }(I=1) \\
\Delta V_{1 / 2}=\text { width of the Na I D line at half-depth; } \\
R V_{1 / 2}=\text { radial velocity of the line at half-depth } \\
W=\text { equivalent width of the line (in mA) } \\
r / r *=\text { extension of the Na I circumstellar cloud, expressed in radii of the central star } \\
\text { The underlined stars are those which present a circumstellar absorption on the Ca II K photspheric } \\
\text { line (see Table 4). }\end{array}$} \\
\hline
\end{tabular}

Gray R.O., 1988, AJ 95, 220

Gray R.O., Garrison R.F., 1989a, ApJS 69, 301

Gray R.O., Garrison R.F., 1989b, ApJS 70, 623

Hauck B., 1978, A\&A 63, 273

Hauck B., 1985, in Calibration of Fundamental Stellar Quantities, Hayes D.S. et al. (eds.), 271-284

Hauck B., Slettebak A., 1989, A\&A 214, 153

Hauck B., 1994, in The MK Process at Fifty Years, Corbally C. \& Garrison R.F. (eds.), ASP Conf. Ser. 60, p. 157

Hauck B., Ballereau D., Chauville J., 1995, A\&AS 109, 505

Hauck B., Künzli M., 1996, Baltic Astr. 5, 303

Higurashi T., Hirata R., 1978, PASJ 30, 615

Hoffleit D., Jaschek C., 1982, The Bright Star Catalogue, Yale Univ. Obs., New Haven

Holweger H., Rentzsch-Holm I., 1995, A\&A 303, 819

Iliev I.K., Barzova I.S., 1995, A\&A 302, 735

Jaschek M., Andrillat Y., Jaschek C., 1991, A\&A 250, 127

King J.R., 1994, MNRAS 269, 209

Künzli M., North P., Kurucz R.L., Nicolet B., 1997, A\&AS
122,51

Kuschnig R., Paunzen E., Weiss W.W., 1994, IBVS 4069, 1 Kuschnig R., Paunzen E., Weiss W.W., 1994, IBVS 4070, 1 Levato H., Abt H.A., 1978, PASP 90, 429

Osawa K., 1965, Ann. Tokyo Astron. Obs. 2nd Ser. IX, 123

Parenago P.P., 1958, Soviet Astron. 2, 151

Paunzen E., 1995, IBVS 4254, 1

Paunzen E., Duffee B., 1995, IBVS 4255, 1

Paunzen E., Heiter U., Weiss W.W., 1995, IBVS 4191, 1

Paunzen E., Weiss W.W., Heiter U., North P., 1997, A\&AS 123, 93

Peniche R., Peña J.H., 1981, PASP 93, 735

Renson P., 1992, Bull. CDS 40, 97

Sadakane K., Nishida M., 1986, PASP 266, 479

Sturenburg S., 1993, A\&A 277, 139

Venn K.A., Lambert D.L., 1990, ApJ 363, 234

Weiss W.W., Paunzen E., Kuschnig R., Schneider H., 1994, A\&A 281, 797 


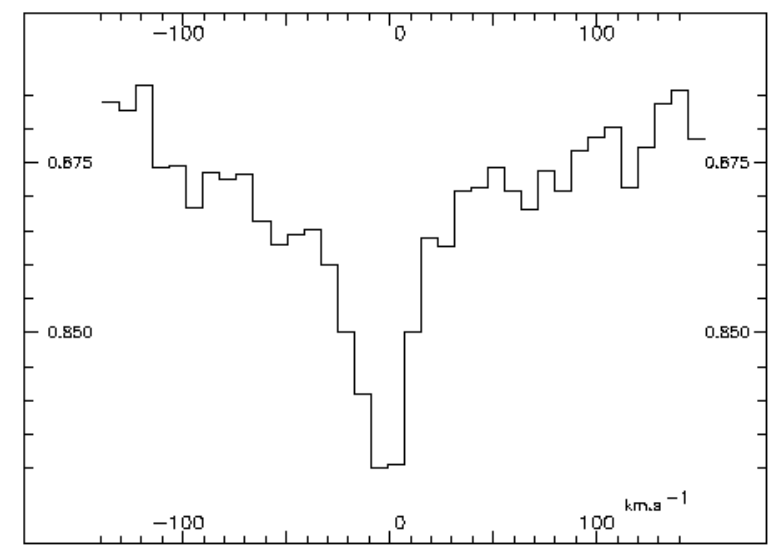

Fig. 1. a) CaII-K line profile for HD 2904

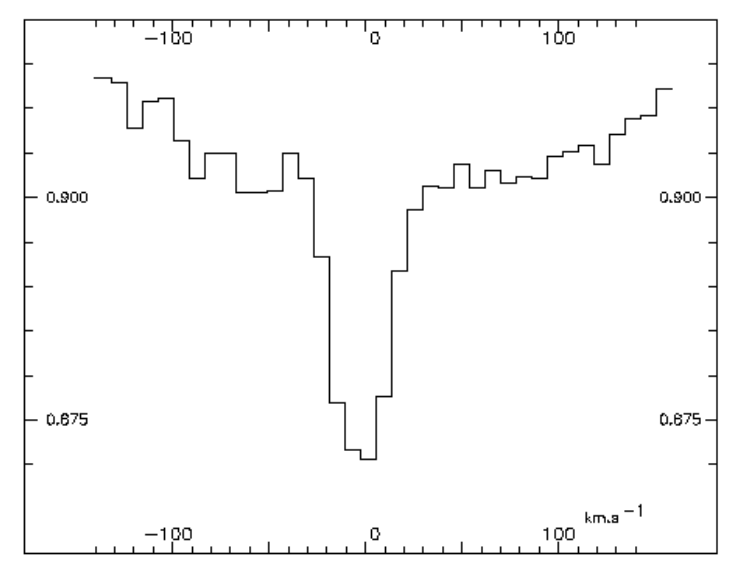

Fig. 1. b) As Fig. 1a for HD 5789

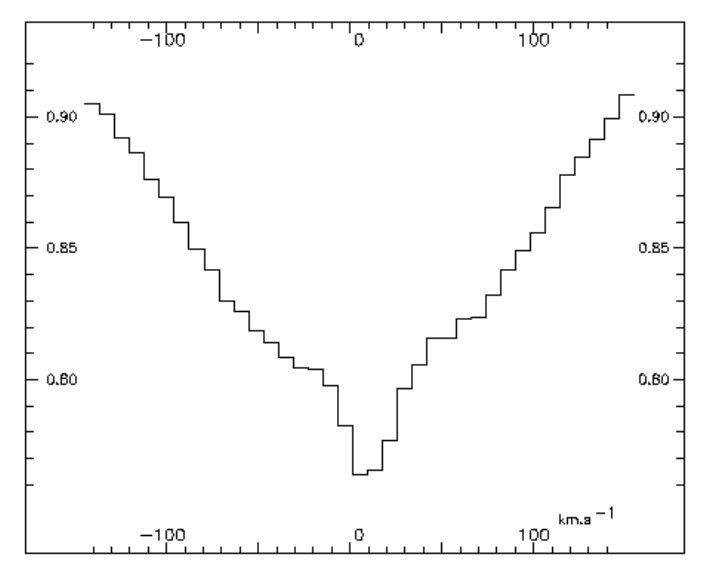

Fig. 1. c) As Fig. 1a for HD 16811

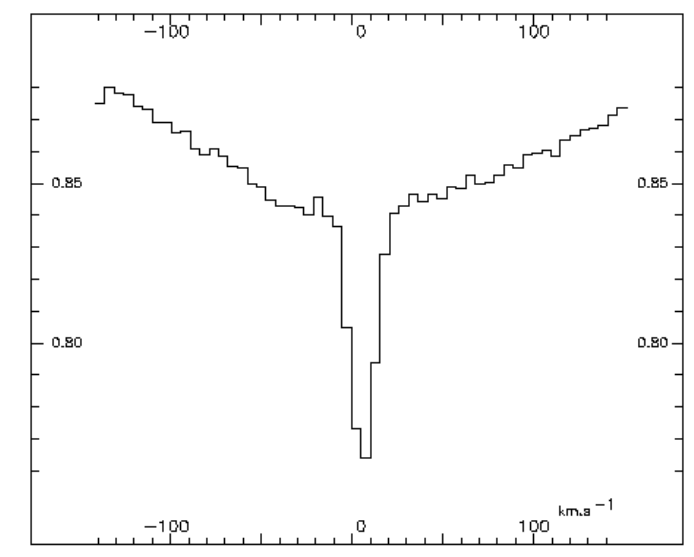

Fig. 1. d) As Fig. 1a for HD 34787 11/11/94

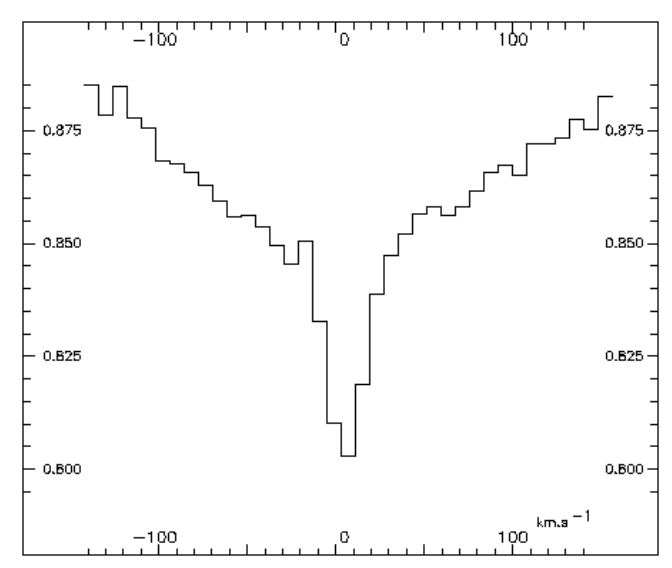

Fig. 1. e) As Fig. 1a for HD 34787 2/11/95

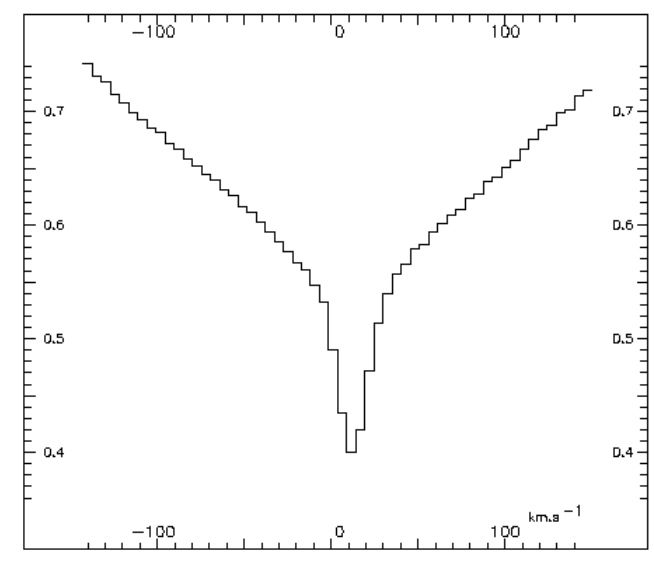

Fig. 1. f) As Fig. 1a for HD 38545 


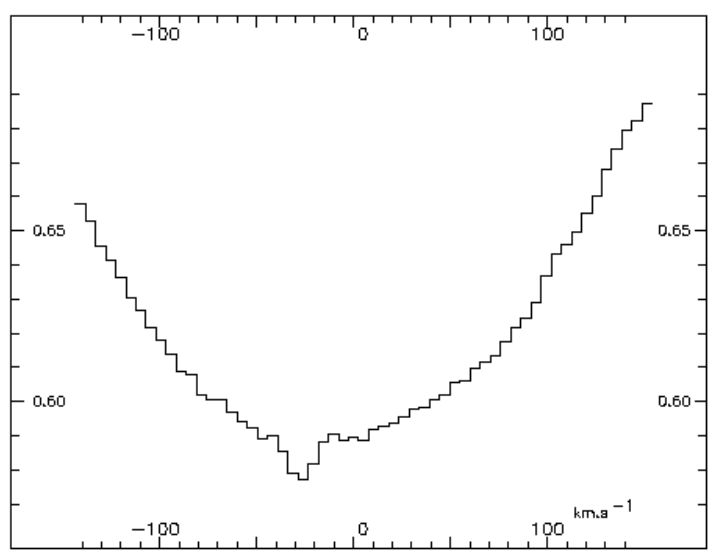

Fig. 1. g) As Fig. 1a for HD 141851

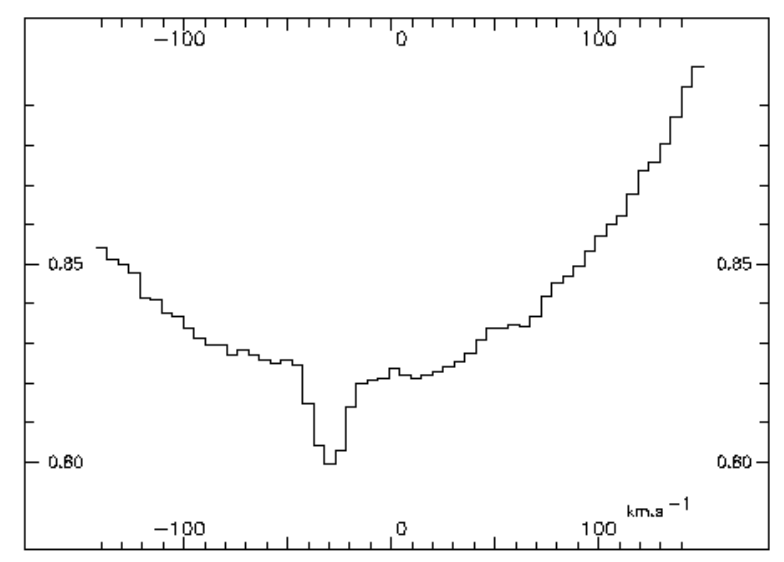

Fig. 1. h) As Fig. 1a for HD 161868

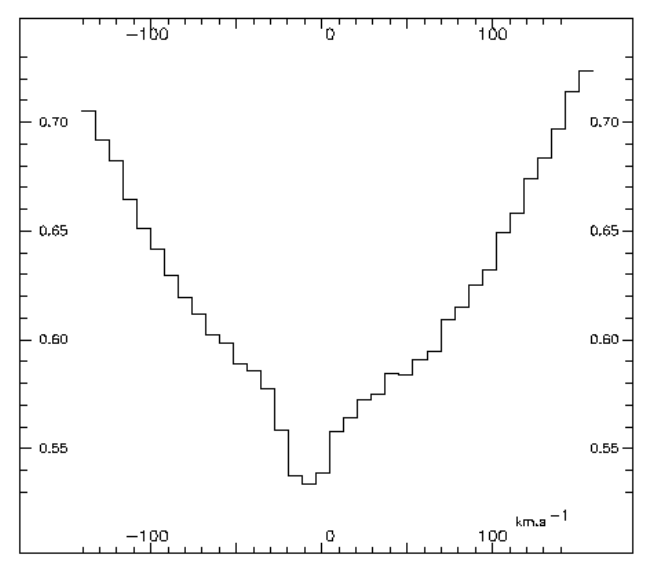

Fig. 1. i) As Fig. 1a for HD 217782

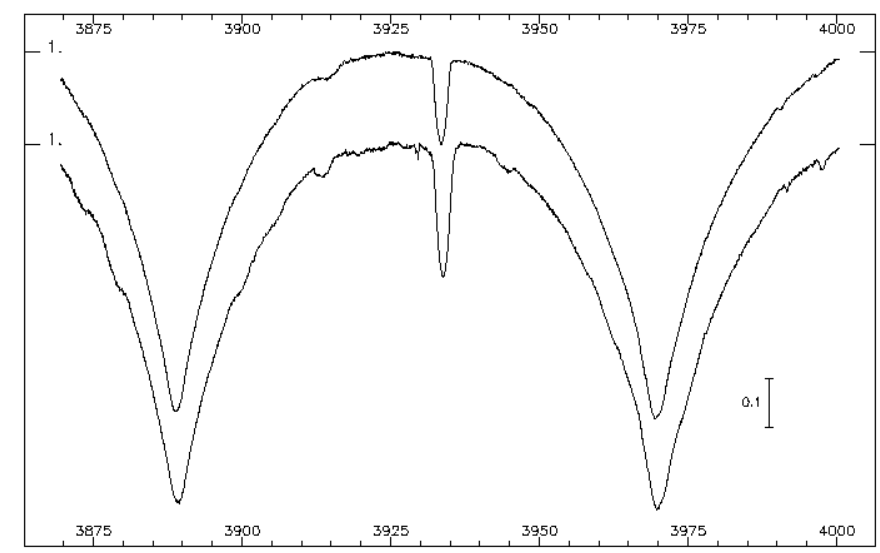

Fig. 2. Comparison of the spectrum of $\lambda$ Boo (upper spectrum) and $\pi^{1}$ Ori mear CaII-K

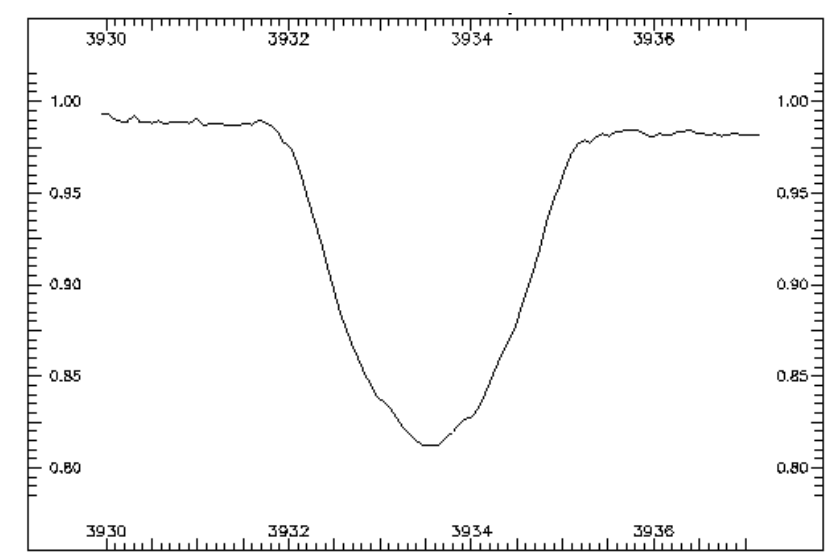

Fig. 3. CaII-K line profile of $\lambda$ Boo

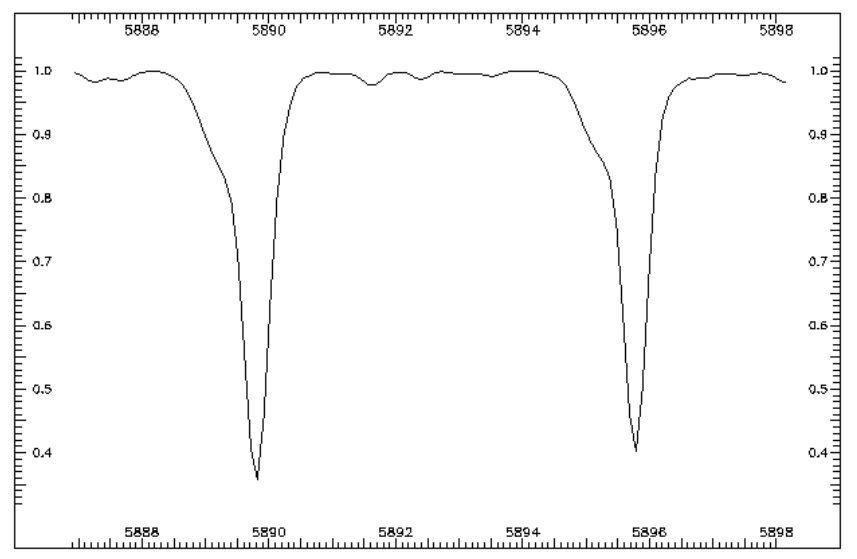

Fig. 4. Spectrum of HD 225180 near the NaI doublet 